\title{
Impulsive observer-based control for linear systems using irregularly sampled measurements
}

\author{
Y. Khaled ${ }^{1,2}$, J-P. Barbot ${ }^{1,3}$, K. Busawon ${ }^{4}$ and D. Benmerzouk ${ }^{2}$ \\ ${ }^{1}$ ECS-Lab ENSEA, 6 Avenue du Ponceau, 95014 Cergy-Pontoise, France \\ ${ }^{2}$ Department of Mathematics, Tlemcen University, Algeria \\ ${ }^{3}$ EPI Non-A, INRIA Lille, Nord-Europe \\ ${ }^{4}$ Northumbria University, Faculty of Engineering and Environment, Newcastle upon Tyne, UK \\ Email: krishna.busawon@unn.ac.uk
}

\begin{abstract}
In this paper, we consider the issue of stabilizing a class of linear systems using irregular sampled output measurements. For this purpose, we design a standard linear state feedback controller and an impulsive observer to provide an estimate the non-measured states, which are subsequently fed back in the control algorithm. We consider linear systems that can be decomposed, via a change of coordinates, into their respective measured and unmeasured dynamics. We consider the two cases whereby the unmeasured subspace is stable and unstable respectively. In the case where the unmeasured subspace is stable, we employ a standard impulsive observer coupled with a continuous linear feedback control. Next, when the unmeasured subspace is unstable, we employ two cascaded observers - an impulsive and a Luenberger observer - in conjunction with a linear feedback control. In order to prove the stability of the overall closed-loop system we proposed a practical stability result for a class of linear impulsive systems. Some simulation results are presented to show the performance of the observer-based control. Finally, some conclusions are drawn.
\end{abstract}

Index Terms-observer-based control, discrete measurement, impulsive systems

\section{INTRODUCTION}

In many practical control applications, we are faced with the issue of controlling a continuous-time system with measurements available at discrete instants of time. When faced with such a situation, one can attempt to discretise the system if the output is sampled fast enough; so that the discretised differential equation describing the system gives a good representation of the original continuous system. One can then employ a discrete output feedback controller or a discrete observer-based controller to control the system. However, in the case where the measurements are irregularly spaced such discretisation is not possible. Such is the case for chemical processes in general, whereby the output measurements are available in discrete time and the time intervals between the samples are not necessarily regularly spaced or constant. In such a case, one has to find alternative means to control these systems.

In this paper, we propose an observer-based control design methodology for a class of linear systems with irregularly sampled output measurements. More precisely, we consider the following class of systems:

$$
\left\{\begin{array}{c}
\dot{x}(t)=F x(t)+G u(t) \\
y\left(t_{k}\right)=H x\left(t_{k}\right)
\end{array}\right.
$$

where $x(t) \in \mathbb{R}^{n}$ is the state, $u(t) \in \mathbb{R}^{m}$ is the input and $y\left(t_{k}\right) \in \mathbb{R}^{p}$ is the output. The matrices $F, G$ and $H$ are constant and of appropriate dimension. Moreover, the system is assumed to be detectable and stabilizable. We assume that there exists a linear transformation $z=T x$ that permits to transform system (1), into the following system:

$$
\left\{\begin{array}{l}
\dot{z}_{1}(t)=A_{11} z_{1}(t)+A_{12} z_{2}(t)+B_{1} u(t) \\
\dot{z}_{2}(t)=A_{22} z_{2}(t)+B_{2} u(t) \\
y\left(t_{k}\right)=z_{1}\left(t_{k}\right)
\end{array}\right.
$$

where $z(t)=\left(z_{1}^{T}, z_{2}^{T}\right)^{T}$ with $A_{11} \in \mathbb{R}^{p \times p}, A_{12} \in \mathbb{R}^{p \times(n-p)}$, $A_{22} \in \mathbb{R}^{(n-p) \times(n-p)}, B_{1} \in \mathbb{R}^{p \times m}, B_{2} \in \mathbb{R}^{(n-p) \times m}$ and $A_{22}$ Hurwitz continuous.

We consider the two cases where the matrix $A_{22}$ is stable and unstable respectively. In the case where $A_{22}$ is stable, we design an impulsive observer, which is basically a copy of the system with an appropriate reset function. Additionally, we employ a continuous linear feedback in order to design an observer-based control for the system. This effectively shows that a separation principle is possible in that case. In the case where $A_{22}$ is not stable the observer design is not trivial. For this, we employ two cascaded observers composed of a Luenberger and an impulsive observer. The reason for including a Luenberger observer is to able to modify the eigenvalues of $A_{22}$ via its output correction term.

The result obtained is also valid in the case where output sampling frequency is smaller than the Nyquist-Shannon sampling frequency; i.e. in the case of sparse measurements (see eg. [1] [2]). Effectively, this work shows that it is possible to guaranty closed loop stability under sparse measurement (see e.g. [3]).

The paper is organized as follows: In the next section a stability result with respect to impulsive systems is presented.This stability result is then used in the subsequent sections to prove the stability of the overall closed-loop system. Additional results on different types of stability of impulsive systems can 
be found in [4], [5], [6], [7] and [2]. In Section 3, an observerbased control is proposed for the case where the unmeasured subspace is stable; i.e. $A_{22}$ is stable. The case where the unmeasured subspace is unstable is considered in Section 4. In Section 5, an example is given in order to highlight the efficiency of the proposed observer based control scheme. Finally, the paper ends with some conclusions and perspectives for future research work.

\section{PRACTICAL STABility FOR A CLASS OF IMPUlsive}

\section{SYSTEM}

In this section, we are going to give some conditions under which a class of linear impulsive systems is practically stable. More precisely, we consider the following class of linear impulsive systems:

$$
\left\{\begin{array}{l}
\dot{x}_{1}(t)=A_{11} x_{1}(t)+A_{12} x_{2}(t) ; \quad t \neq t_{k} \\
\dot{x}_{2}(t)=A_{22} x_{2}(t) \\
x_{1}\left(t_{k}^{+}\right)=R x_{1}\left(t_{k}\right) \\
x_{2}\left(t_{k}^{+}\right)=x_{2}\left(t_{k}\right)
\end{array}\right.
$$

where $x(t) \in \mathbb{R}^{n}, A_{11} \in \mathbb{R}^{p \times p}, A_{12} \in \mathbb{R}^{p \times(n-p)}, A_{22} \in$ $\mathbb{R}^{(n-p) \times(n-p)}$ and $R \in \mathbb{R}^{p \times p}$. The sampling sequence $t_{k} \in$ $T=\left\{t_{i}: i \in \mathbb{N}\right\} \subset \mathbb{R}$. We assume that there exists $\tau_{\max }$ and $\tau_{\min }$ with $\tau_{\max }>\tau_{\min }>0$ such that $\forall i>0$

$$
t_{i}+\tau_{\min }<t_{i+1}<t_{i}+\tau_{\max } .
$$

The above strict inequality permits to exclude the Zeno phenomena since system (3) is essentially a particular class of hybrid systems [8].

Additionally, we define:

$$
\begin{aligned}
& x\left(t_{k}^{+}\right) \triangleq \lim _{h \rightarrow 0} x\left(t_{k}+h\right) \\
& x\left(t_{k}^{-}\right) \triangleq \lim _{h \rightarrow 0} x\left(t_{k}-h\right)=x\left(t_{k}\right)
\end{aligned}
$$

Throughout the paper, we assume that the system (3) verifies the sufficient conditions for the existence of solution [7].

Before stating the main stability result of this section, we recall some important results and definitions. First, recall that if $A \in \mathbb{R}^{n \times n}$ is a discrete Hurwitz matrix (that is, all eigenvalues are strictly inside the unit circle of the complex plane) then $\lim _{k \rightarrow \infty} A^{k}=0$. Additionally, there exist at least a matrix norm $\|\bullet\|$, such that $\|A\|<1$.

Next, we recall that:

Definition 1. [4] The system (3) is said to be:

1) Practically stable with respect to $(\delta, \epsilon)$ if given $(\delta, \epsilon)$ with $0<\delta<\epsilon$, we have that $\left\|x\left(t_{0}\right)\right\|<\delta$ implies $\|x(t)\|<\epsilon, t>t_{0}$ for some $t_{0} \in R$.

2) Uniformly practically stable with respect to $(\delta, \epsilon)$, if 1$)$ holds for every $t_{0} \in R$.

3) Practically asymptotically stable with respect to $(\delta, \epsilon)$, if 1) holds and $\lim _{t \rightarrow \infty}\|x(t)\|=0$.

We can now state the following:

Theorem 1. Assume that system (3) verifies the following conditions:
1) $A_{22}$ is a continuous Hurwitz matrix; that is its eigenvalues have strictly negative real part.

2) $\left\|R e^{A_{11} t}\right\|^{2}<1$, for all $t \in\left[0, \tau_{\max }[>0\right.$.

Then, $\forall \epsilon>0$, the state of system (3) converges to a ball of radius $\epsilon$ (practical stability).

Proof: First, the solution of (3) for $t \in\left[t_{k}^{+}, t_{k+1}^{-}\right]$with the initial time $t_{0}=t_{k}^{+}$is given by

$$
x_{1}(t)=e^{A_{11}\left(t-t_{k}\right)} x_{1}\left(t_{k}^{+}\right)+\int_{t_{k}}^{t} e^{A_{11}(t-\tau)} A_{12} x_{2}(\tau) d \tau
$$

and

$$
x_{2}(t)=e^{A_{22}\left(t-t_{k}\right)} x_{2}\left(t_{k}^{+}\right)
$$

Replacing (6) in (5), we obtain, at $t=t_{k+1}$ :

$$
\begin{aligned}
x_{1}\left(t_{k+1}\right)= & e^{A_{11} \theta_{k}} x_{1}\left(t_{k}^{+}\right) \\
& +\int_{t_{k}}^{t_{k+1}} e^{A_{11}\left(t_{k+1}-\tau\right)} A_{12} e^{A_{22}\left(\tau-t_{k}\right)} x_{2}\left(t_{k}^{+}\right) d \tau
\end{aligned}
$$

where $\theta_{k}=t_{k+1}-t_{k}$.

Consider the following Lyapunov function:

$$
V(x)=V_{1}\left(x_{1}\right)+V_{2}\left(x_{2}\right)
$$

with

$$
\begin{aligned}
& V_{1}\left(x_{1}\right)=x_{1}^{T} x_{1}=\left\|x_{1}\right\|^{2} \\
& V_{2}\left(x_{2}\right)=x_{2}^{T} x_{2}=\left\|x_{2}\right\|^{2} .
\end{aligned}
$$

Starting with $V_{2}\left(x_{2}\right)$, we get

$$
\begin{aligned}
\triangle V_{2}\left(x_{2}\left(t_{k}\right)\right) & =V_{2}\left(x_{2}\left(t_{k+1}^{+}\right)\right)-V_{2}\left(x_{2}\left(t_{k}^{+}\right)\right) \\
& =V_{2}\left(x_{2}\left(t_{k+1}\right)\right)-V_{2}\left(x_{2}\left(t_{k}^{+}\right)\right) \\
& =\left\|e^{A_{22} \theta_{k}} x_{2}\left(t_{k}^{+}\right)\right\|^{2}-\left\|x_{2}\left(t_{k}^{+}\right)\right\|^{2} \\
& \leq\left(\left\|e^{A_{22} \theta_{k}}\right\|^{2}-1\right) V\left(x_{2}\left(t_{k}^{+}\right)\right)
\end{aligned}
$$

Since the matrix $A_{22}$ is Hurwitz continuous, this implies that $e^{A_{22} \theta_{k}}$ is a discrete Hurwitz matrix and therefore

$$
\left\|e^{A_{22} \theta_{k}}\right\|^{2}<1
$$

Consequently,

$$
\triangle V_{2}\left(x_{2}\left(t_{k}\right)\right)<0
$$

This inequality ensures the uniform asymptotic convergence of $x_{2}$ to zero (uniformly with respect to $x_{1}$ ).

Therefore, $\forall \delta>0$ there exists $h$ such that:

$$
\forall k>h, \quad x_{2}\left(t_{k}\right)<\delta \text {. }
$$

Now, for the Lyapunov function $V_{1}$, we have

$$
\begin{aligned}
\triangle V_{1}\left(x_{1}\left(t_{k}\right)\right) & =V_{1}\left(x\left(t_{k+1}^{+}\right)\right)-V_{1}\left(x\left(t_{k}^{+}\right)\right) \\
& =V_{1}\left(R x_{1}\left(t_{k+1}\right)\right)-V_{1}\left(x_{1}\left(t_{k}^{+}\right)\right)
\end{aligned}
$$

By replacing the above expression of $x_{1}\left(t_{k+1}\right)$, we get:

$$
\begin{aligned}
\triangle V_{1} & \leq\left\|R e^{A_{11} \theta_{k}} x_{1}\right\|^{2}-\left\|x_{1}\right\|^{2} \\
& +\left\|R \int_{t_{k}}^{t_{k+1}} e^{A_{11}\left(t_{k+1}-\tau\right)} A_{12} e^{A_{22}\left(\tau-t_{k}\right)} d \tau x_{2}\right\|^{2} \\
& \leq\left(\left\|R e^{A_{11} \theta_{k}}\right\|^{2}-1\right) V_{1}\left(x_{1}\right) \\
+ & \left\|A_{12}\right\|^{2}\left\|x_{2}\right\|^{2} \int_{t_{k}}^{t_{k+1}}\left\|R e^{A_{11}\left(t_{k+1}-\tau\right)}\right\|^{2}\left\|e^{A_{22}\left(\tau-t_{k}\right)}\right\|^{2} d \tau
\end{aligned}
$$


Since $A_{22}$ is Huzwitz continuous and that from the second condition $\left\|R e^{A_{11}\left(t_{k+1}-\tau\right)}\right\|^{2}<1$ for all $\tau \in\left[t_{k}, t_{k+1}[\right.$, we have:

$$
\begin{aligned}
\triangle V_{1} & \leq\left(\left\|R e^{A_{11} \theta_{k}}\right\|^{2}-1\right) V_{1}\left(x_{1}\right)+\left\|A_{12}\right\|^{2}\left\|x_{2}\right\|^{2} \int_{t_{k}}^{t_{k+1}} d \tau \\
& \leq\left(\left\|R e^{A_{11} \theta_{k}}\right\|^{2}-1\right) V_{1}\left(x_{1}\right)+\theta_{k}\left\|A_{12}\right\|^{2}\left\|x_{2}\right\|^{2}
\end{aligned}
$$

So, from (8) we have

$\forall \delta>0, \exists h>0, \forall k>h:\left\|x_{2}\left(t_{k}\right)\right\|<\delta$.

Now, choose $\epsilon>0$ and define $V_{1, \min }=\min _{\left\|x_{1}\right\|=\epsilon} V_{1}\left(x_{1}\right)$. Then, for all $\left\|x_{1}(k)\right\|>\epsilon$ and $\theta_{k}<\frac{\left(\left\|R e^{A_{11} \theta_{k}}\right\|^{2}-1\right) V_{1 \mathrm{~min}}}{\left\|A_{12}\right\| \delta}$, we have:

$$
\triangle V_{1}\left(x_{1}\left(t_{k}\right)\right)<0
$$

Owing to the fact that $\delta>0$ can be arbitrarily chosen, the only constraint on $\theta_{k}$ is $\left\|R e^{A_{11} \theta_{k}}\right\|^{2}<1$. Consequently (7) and the previous inequality imply that $\exists h$ such that $\forall k>h$ we have $\triangle V\left(x_{1}\left(t_{k}\right), x_{2}\left(t_{k}\right)\right)<0$ if $\left\|x_{1}\right\|>\epsilon$.

This completes the proof of Theorem 1 .

\section{CASE 1: STABLE UNMEASURED SUBSPACE}

Consider system (2) and assume that the matrix $A_{22}$ is Hurwitz continuous.

In a compact form system (2) is written as:

$$
\left\{\begin{array}{l}
\dot{z}(t)=A z(t)+B u(t) \\
y\left(t_{k}\right)=C z\left(t_{k}\right)
\end{array}\right.
$$

where $A=\left(\begin{array}{cc}A_{11} & A_{12} \\ 0 & A_{22}\end{array}\right), B=\left(\begin{array}{c}B_{1} \\ B_{2}\end{array}\right)$ and $C=$ $\left(\begin{array}{ll}I_{p} & 0\end{array}\right)$ with $I_{p}$ being the $p$-dimensional identity matrix.

Remark 1: Note that the above assumption that there exists a linear transformation $z=T x$ that permits to transform system (1), into (2) is not too restrictive, because if $\operatorname{ker}\{H\}$ is included on the stable subspace of $F$, it is always possible to select a part of the output $y\left(t_{k}\right)$ as the considered output, in order to obtain the system (2) under the transformation $z=T x$.

We design an observer for system (2) as follows:

$$
\left\{\begin{array}{l}
\dot{\hat{z}}_{1}(t)=A_{11} \hat{z}_{1}(t)+A_{12} \hat{z}_{2}(t)+B_{1} u(t) \\
\dot{z}_{2}(t)=A_{22} \hat{z}_{2}(t)+B_{2} u(t) \\
\hat{z}_{1}\left(t_{k}^{+}\right)=R \hat{z}_{1}\left(t_{k}\right)+\left(I_{p}-R\right) z_{1}\left(t_{k}\right) \\
\hat{y}\left(t_{k}\right)=\hat{z}_{1}\left(t_{k}\right)
\end{array}\right.
$$

where $R \in \mathbb{R}^{m \times n}$ is the reset matrix, such that $R=\operatorname{diag}\left\{r_{1}, . ., r_{p}\right\}$, with $\left.r_{i} \in\right]-1,1[$, for $i=1, . ., p$.

As a result, a linear observer based feedback is given by:

$$
u(t)=-K \hat{z} \triangleq\left(-K_{1},-K_{2}\right)\left(\begin{array}{c}
\hat{z}_{1}(t) \\
\hat{z}_{2}(t)
\end{array}\right)
$$

where $K \in \mathbb{R}^{m \times n}$ is chosen such that $(A-B K)$ is stable.

Theorem 2. Assume that there exists $\tau_{\max }$ and $\tau_{\min }$ with $\tau_{\max }>\tau_{\min }>0$ such that $\forall i>0, t_{i}+\tau_{\min }<t_{i+1}<$ $t_{i}+\tau_{\max }$. Then, the closed-loop system:

$$
\dot{z}(t)=A z(t)-B K \hat{z}(t)=(A-B K) z(t)+B K e(t)
$$

is practically asymptotically stable.

Proof: By setting $e_{1}=z_{1}-\hat{z}_{1}, e_{2}=z_{2}-\hat{z}_{2}$, we obtain:

$$
\left(\begin{array}{c}
\dot{e}_{1} \\
\dot{e}_{2}
\end{array}\right)=\left(\begin{array}{cc}
A_{11} & A_{12} \\
0 & A_{22}
\end{array}\right)\left(\begin{array}{c}
e_{1} \\
e_{2}
\end{array}\right)
$$

On the other hand, by expanding the closed-loop system (12) we obtain

$$
\dot{z}=(A-B K) z+B K e
$$

where

$$
\begin{aligned}
& A_{p p}=(A-B K)=\left(\begin{array}{cc}
A_{11}-B_{1} K_{1} & A_{12}-B_{1} K_{2} \\
-B_{2} K_{1} & A_{22}-B_{2} K_{2}
\end{array}\right) \\
& B_{p p}=B K=\left(\begin{array}{cc}
B_{2} K_{1} & B_{1} K_{2} \\
B_{2} K_{1} & B_{2} K_{2}
\end{array}\right)
\end{aligned}
$$

The reset function becomes:

$$
e_{1}\left(t_{k}^{+}\right)=R e_{1}\left(t_{k}\right)
$$

The overall state dynamics and observation error dynamics can be written as

$$
\left(\begin{array}{c}
\dot{e} \\
\dot{z}
\end{array}\right)=\left(\begin{array}{l|l}
A & 0 \\
\hline B_{p p} & A_{p p}
\end{array}\right)\left(\begin{array}{c}
e \\
z
\end{array}\right)
$$

with the following reset function:

$$
\left[\begin{array}{l}
e_{1}\left(t_{k}^{+}\right) \\
e_{2}\left(t_{k}^{+}\right) \\
z_{1}\left(t_{k}^{+}\right) \\
z_{2}\left(t_{k}^{+}\right)
\end{array}\right]=\left[\begin{array}{cc}
R & 0 \\
0 & I_{2 n-p}
\end{array}\right]\left[\begin{array}{l}
e_{1}\left(t_{k}\right) \\
e_{2}\left(t_{k}\right) \\
z_{1}\left(t_{k}\right) \\
z_{2}\left(t_{k}\right)
\end{array}\right]
$$

The observation error dynamics has exactly the same form as system (3) and is independent of $z_{i}$, so $e$ is practically asymptotically stable. Now, again from the theorem 1 for all $\epsilon>0 \exists h>0$ such that $\left\|e_{i}(k)\right\|<\epsilon$. Now setting $V_{3}(z) \triangleq$ $z^{T} z=\|z\|^{2}$ and proceeding in the same way as for the proof of Theorem 1, we obtain:

$$
\begin{aligned}
\triangle V_{3}\left(z\left(t_{k}\right)\right)= & V_{3}\left(e^{A_{p p} \theta_{k}} z\left(t_{k}\right)\right. \\
& \left.+\int_{t_{k}}^{t_{k+1}} e^{A_{p p}\left(t_{k+1}-\tau\right)} B_{p p} e(\tau) d \tau\right) \\
& -V_{3}\left(x_{1}\left(t_{k}\right)\right)
\end{aligned}
$$

As $k>h:\|e\|^{2}<\epsilon$, we have:

$$
\begin{aligned}
\triangle V_{3} \leq & \left\|e^{A_{p p} \theta_{k}} z\right\|^{2} \\
& +\left\|\int_{t_{k}}^{t_{k+1}} e^{A_{p p}\left(t_{k+1}-\tau\right)} B_{p p} d \tau \epsilon\right\|^{2}-\|z\|^{2} \\
\leq & \left(\left\|R e^{A_{p p} \theta_{k}}\right\|^{2}-1\right) V_{3}\left(x_{1}\right) \\
& +\int_{t_{k}}^{t_{k+1}}\left\|R e^{A_{p p}\left(t_{k+1}-\tau\right)} B_{p p} e^{A_{22}\left(\tau-t_{k}\right)}\right\|^{2} d \tau\left\|x_{2}\right\|^{2} \\
\leq & \left(\left\|e^{A_{p p} \theta_{k}}\right\|^{2}-1\right) V_{3}(z(k)) \\
& +\left\|B_{p p}\right\|^{2} \epsilon \int_{t_{k}}^{t_{k+1}}\left\|e^{A_{p p}\left(t_{k+1}-\tau\right)}\right\|^{2} d \tau
\end{aligned}
$$

This ensures the practical stability and completes the proof of Theorem 2. 


\section{CASE 2: unstable unMeasured Subspace}

Consider again system (2) and assume that the matrix $A_{22}$ is unstable.

Remark 2. As in the previous section, that there exists a linear transformation $z=T x$ that permits to transform system (1), into (2) is not too restrictive in this case either. This is because it is always possible to consider only one part of the output $y$ as a considered output, in order to obtain the system (2) under the transformation $z=T x$. However, in some cases, this output restriction can destroy the system detectability and then the basic assumption for considering only detectable and stabilizable system is violated.

The observer-based control for system (2) is quite different to the one proposed in the previous section as far as the observer part is concerned. In fact, we employ two cascaded observers - an impulsive and a Luenberger observer. More precisely,

$$
\left\{\begin{array}{l}
\dot{\hat{z}}_{1}(t)=A_{11} \hat{z}_{1}(t)+A_{12} \hat{z}_{2}(t)+B_{1} u(t) \\
\dot{z}_{2}(t)=A_{22} \hat{z}_{2}(t)+M\left(\xi_{2}(t)-\hat{z}_{2}(t)\right)+B_{2} u(t) \\
\dot{\xi}_{1}(t)=A_{11} \xi_{1}(t)+A_{12} \xi_{2}(t)+L_{1}\left(\hat{z}_{1}(t)-\xi_{1}(t)\right)+B_{1} u(t) \\
\dot{\xi}_{2}(t)=A_{22} \xi_{2}(t)+L_{2}\left(\hat{z}_{1}(t)-\xi_{1}(t)\right)+B_{2} u(t) \\
\hat{z}_{1}\left(t_{k}^{+}\right)=\bar{R} \hat{z}_{1}\left(t_{k}\right)+\left(I_{d}-\bar{R}\right) z_{1}\left(t_{k}\right)
\end{array}\right.
$$

where $K \in \mathbb{R}^{m \times n}$, with $\bar{R}=\operatorname{diag}\left\{\bar{r}_{1}, . ., \bar{r}_{n_{1}}\right\}$ and $-1<$ $\bar{r}_{i}<1$, for $i=1, \ldots, n_{1}$ and $M, L_{1}$ and $L_{2}$ are matrices of appropriate dimensions.

As for the controller, we employ a linear control as before:

$$
u(t)=-K \hat{z} \triangleq\left(K_{1}, K_{2}\right)\left(\begin{array}{c}
\hat{z}_{1}(t) \\
\hat{z}_{2}(t)
\end{array}\right)
$$

whereby the estimated state variables $\hat{z}_{1}$ and $\hat{z}_{2}$ are issued from the generalized cascaded impulsive observer.

Denote $\chi \triangleq\left(e_{1}^{T}, e_{2}^{T}, \varepsilon_{1}^{T}, \varepsilon_{2}^{T}, z_{1}^{T}, z_{2}^{T}\right)^{T}$ with $e_{i}=z_{i}-\hat{z}_{i}$ and $\varepsilon_{i}=z-\xi$. Then, one can show that:

$$
\left(\begin{array}{c}
\dot{e}_{1} \\
\dot{e}_{2}
\end{array}\right)=\left(\begin{array}{cc}
A_{11} & A_{12} \\
0 & A_{22}-M
\end{array}\right)\left(\begin{array}{l}
e_{1} \\
e_{2}
\end{array}\right)+\left(\begin{array}{c}
0 \\
M \varepsilon_{2}
\end{array}\right)
$$

and

$$
\left(\begin{array}{c}
\dot{\varepsilon}_{1} \\
\dot{\varepsilon}_{2}
\end{array}\right)=\left(\begin{array}{cc}
A_{11}-L_{1} C & A_{12} \\
-L_{2} C & A_{22}
\end{array}\right)\left(\begin{array}{c}
\varepsilon_{1} \\
\varepsilon_{2}
\end{array}\right)+\left(\begin{array}{c}
L_{1} e_{1} \\
L_{2} e_{1}
\end{array}\right)
$$

Additionally, the the closed-loop system via the observer is given by:

$$
\begin{aligned}
\left(\begin{array}{c}
\dot{z}_{1} \\
\dot{z}_{2}
\end{array}\right)= & \left(\begin{array}{cc}
B_{2} K_{1} & B_{1} K_{2} \\
B_{2} K_{1} & B_{2} K_{2}
\end{array}\right)\left(\begin{array}{c}
e_{1} \\
e_{2}
\end{array}\right) \\
& +\left(\begin{array}{cc}
A_{11}-B_{1} K_{1} & A_{12}-B_{1} K_{2} \\
-B_{2} K_{1} & A_{22}-B_{2} K_{2}
\end{array}\right)\left(\begin{array}{c}
z_{1} \\
z_{2}
\end{array}\right)
\end{aligned}
$$

We are now able to state the main result of this section:

Theorem 3. Assume that system (2) satisfies Assumption 2 and consider the closed-loop system (15). Then, for all $\beta_{\max }>0$ and $\epsilon>0$ with $\beta_{\max }>\epsilon$, there exist a maximum sampling period $\theta_{\max }>0$ and at least a reset matrix $R$ such that for any initial condition $\chi(0)$ such that $\|\chi(0)\|_{\epsilon}<\beta_{\max }$, the original system's state $z$ converges to $\|z\|<\frac{\epsilon}{2}$ and the extended state at each sampling time $t_{k}^{+}$converge into a set $B_{\epsilon} \triangleq\{\chi:\|\chi\|<\epsilon\}$, if the following conditions are verified:

i) the matrix $\Gamma_{1}=\left(\begin{array}{ccc}A_{22}-M & 0 & M \\ 0 & A_{11}-L_{1} & A_{12} \\ 0 & -L_{2} & A_{22}\end{array}\right)$ is a continuous Hurwitz matrix. ii) $\left.\begin{array}{cc}\text { the } & \text { control } \\ A_{11}-B_{1} K_{1} & A_{12}-B_{1} K_{2} \\ -B_{2} K_{1} & A_{22}-B_{2} K_{2}\end{array}\right)$ is atrix $\quad \Gamma_{2}$ a continuous Hurwitz
matrix.

Proof: One can check that

$$
e_{1}\left(t_{k}^{+}\right)=R e_{1}\left(t_{k}\right)
$$

Now, we define a Lyapunov function $V_{1}\left(e_{1}\right) \triangleq e_{1}^{T} e_{1}=\left\|e_{1}\right\|^{2}$ , we obtain

$$
\begin{aligned}
& V_{1}\left(e_{1}\left(t_{k+1}^{+}\right)\right) \\
& \quad=V_{1}\left(R e^{A_{11} \theta_{k}} e_{1}\left(t_{k}^{+}\right)+\int_{t_{k}}^{t_{k+1}} e^{A_{11}\left(t_{k+1}-\tau\right)} A_{12} e_{2}(\tau) d \tau\right)
\end{aligned}
$$

Assuming that $\forall \tau \in\left[t_{k}^{+}, t_{k+1}^{+}\left[,\left\|e_{2}(\tau)\right\|<\beta_{\max }\right.\right.$ (this will be proved later in the proof), with the same arguments as for Theorem 1, we have:

$$
\begin{aligned}
& V_{1}\left(e_{1}\left(t_{k+1}^{+}\right)\right) \leq\left\|R e^{A_{11} \theta_{k}} e_{1}\right\|^{2} \\
& \quad \quad+\left\|R \int_{t_{t}}^{t_{k+1}} e^{A_{11}\left(t_{k+1}-\tau\right)} A_{12} e^{A_{22}\left(\tau-t_{k}\right)} \beta_{\max } d \tau\right\|^{2} \\
& \quad \leq\left(\left\|R e^{A_{11} \theta_{k}}\right\|^{2}\right) V_{1, \max } \\
& +\left\|A_{12}\right\|^{2} \beta_{\max } \int_{t_{k}}^{t_{k+1}}\left\|R e^{A_{11}\left(t_{k+1}-\tau\right)}\right\|^{2}\left\|e^{A_{22}\left(\tau-t_{k}\right)}\right\|^{2} d \tau
\end{aligned}
$$

with $V_{1, \max } \triangleq \max _{\left\|e_{1}\right\|=\beta_{\max }}\left\{V_{1}\left(e_{1}\right)\right\}$. It is always possible to find $R$ such that:

$$
V_{1}\left(x_{1}\left(t_{k+1}^{+}\right)\right)<\epsilon^{\prime} \quad \text { or } \quad\left\|\chi\left(t_{k+1}\right)\right\|<\left(\frac{\epsilon}{4}\right)^{2}
$$

Note that the above inequality is verified by $R=0$ and, by continuity, also by a neighborhood of $R=0$. Setting $\chi_{2} \triangleq$ $\left(e_{2}^{T}, \varepsilon_{1}^{T}, \varepsilon_{2}^{T}, z_{1}^{T}, z_{2}^{T}\right)^{T}$ and using the Lyapunov function $V_{2} \triangleq$ $\chi_{2}^{T} P_{2} \chi_{2}$, with $P_{2}$ a symmetric positive definite (SPD) matrix, we have:

$$
\begin{aligned}
\dot{V}_{2}= & {\left[A_{2} \chi_{2}+\bar{B}_{2} e_{1}\right]^{T} P_{2} \chi_{2} } \\
& +\chi_{2}^{T} P_{2}\left[A_{2} \chi_{2}+\bar{B}_{2} e_{1}\right]
\end{aligned}
$$

with

$$
A_{2}=\left[\begin{array}{l|l}
\Gamma_{1} & 0 \\
\hline \Gamma_{3} & \Gamma_{2}
\end{array}\right]
$$

where

$$
\Gamma_{3}=\left(\begin{array}{ccc}
\tilde{B}_{1} K_{2} & 0 & 0 \\
\tilde{B}_{2} K_{2} & 0 & 0
\end{array}\right)
$$

and $\bar{B}_{2}=\left[0^{T}, L_{1}^{T}, L_{2}^{T},\left(B_{1} K_{1}\right)^{T},\left(B_{2} K_{2}\right)^{T}\right]^{T}$. Conditions i) and ii) of Theorem 3 ensure that $A_{2}$ is a continuous Hurwitz matrix. Consequently, there exists an SPD matrix $P_{2}$ such that $A_{2}^{T} P_{2}+P_{2} A_{2}=-I_{d}$. Consequently,

$$
\dot{V}_{2}=-\left\|\chi_{2}\right\|^{2}+2\left\|B_{2}\right\|\left\|e_{1}\right\|\left\|P_{2}\right\|\left\|\chi_{2}\right\|
$$


Considering the fact that $\left\|\chi_{2}\right\| \geq \frac{\epsilon}{2}$ and for $\left\|e_{1}\right\| \leq\left(\frac{\epsilon}{4}\right)^{2}$ by continuity of $e_{1}$ between $\left[t_{k}, t_{k+1}^{-}\right]$, then there exists $\theta_{\max }$ such that $\forall \tau \in\left[t_{k}, t_{k}+\theta_{\max }\right]$, we have:

$$
\dot{V}_{2}=-\left\|\chi_{2}\right\|^{2}+2\left\|B_{2}\right\|\left\|e_{1}\right\|\left\|P_{2}\right\|\left\|\chi_{2}\right\|
$$

Therefore, $X_{2}$ converges to $\frac{\epsilon}{2}$ and $e_{1}\left(t_{k}\right)$ converges to $\left(\frac{\epsilon}{4}\right)^{2}$ and hence $\chi\left(t_{k}\right)$ converges to $\left(\frac{\epsilon}{2}+\frac{\epsilon}{4}\right)^{2}$ and $z$ converges to $\frac{\epsilon}{2}$.

This ends the proof of Theorem 3.

\section{Simulation Results}

Let us consider the following linear triangular system:

$$
\left\{\begin{array}{c}
\dot{x}_{1}=2 x_{1}+x_{2}+3 x_{3}+u \\
\dot{x}_{2}=x_{2}+x_{3} \\
\dot{x}_{3}=-x_{3}-u \\
y\left(t_{k}\right)=x_{1}\left(t_{k}\right)
\end{array}\right.
$$

Here, we have

$$
\begin{aligned}
& A_{11}=2, A_{12}=\left(\begin{array}{ll}
1 & 3
\end{array}\right) \\
& A_{22}=\left(\begin{array}{cc}
1 & 1 \\
0 & -1
\end{array}\right)
\end{aligned}
$$

It can be seen that the matrix $A_{22}$ is unstable. We are therefore in the Case 2 whereby the unmeasured subspace is unstable. The main objective is stabilise system (17) using an observer-based linear control law $u=-k_{1} \hat{x}_{1}-k_{2} \hat{x}_{2}-k_{3} \hat{x}_{3}$.

Here we choose $k_{1}=-48, k_{2}=-30$ and $k_{3}=-55$. In this case, all the eigenvalues will be located at -2 and -1 in the left-half complex plane. We design a cascaded observer of the form (13), by choosing the parameters as follows: For the impulsive observer and in order to ensure the convergence of the observer the observer gains is designed as follows $R=0$, $M=\left(\begin{array}{ll}5 & 0 \\ 0 & 0\end{array}\right), L_{1}=6$ and $L_{2}=\left(\begin{array}{l}6 \\ 0\end{array}\right)$. The parameters of observer control satisfy the conditions of Theorem 3, with the corresponding matrix of observer is

$$
\Gamma_{1}=\left(\begin{array}{ccccc}
-4 & 1 & 0 & 5 & 0 \\
0 & -1 & 0 & 0 & 0 \\
0 & 0 & -4 & 1 & 3 \\
0 & 0 & -6 & 1 & 1 \\
0 & 0 & 0 & 0 & -1
\end{array}\right)
$$

and the corresponding matrix of control is

$$
\Gamma_{2}=\left(\begin{array}{ccc}
-48 & 31 & 58 \\
1 & 1 & 0 \\
-30 & -56 & -48
\end{array}\right)
$$

Fig. 1 and Fig. 2 shows the performance of the observer (13) with two different periods of measure; the first $\theta_{k}=0.4 \mathrm{~s}$ is fixed and the second $\theta_{k} \in[0.3 s, 0.6 s]$ is variable. The initial conditions are chosen as follows: $x(0)=(0.1,-0.1,0.1)$, $\hat{z}(0)=(1,0.6,0.5)$.

Fig 3 show that with a variable sampling period $\theta_{k} \in$ $[0.3 s, 0.6 s]$, the estimated states $\hat{x}_{1}, \hat{x}_{2}$ and $\hat{x}_{3}$ converges
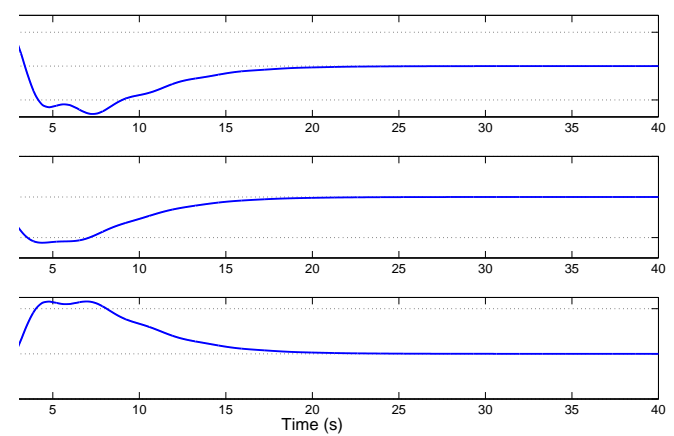

Fig. 1. System states with sampling period $\theta_{k}=0.4$
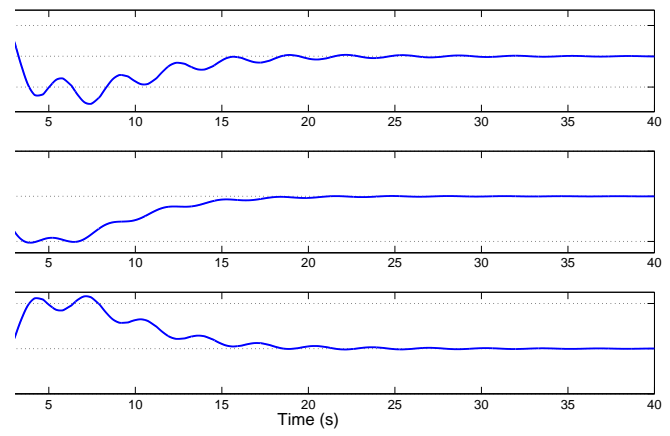

Fig. 2. System states with sampling period $\theta_{k} \in[0.3 s, 0.6 s]$

respectively to the states $x_{1}, x_{2}$ and $x_{3}$ of the system. It should be noted that the coupling matrix $M$, plays a very important role, permitting the stabilisation of the system (17).

\section{CONCLUSION}

In this paper, we have shown that it is possible to design an observer-based control for a class of linear systems using discrete measurements with a variable sampling period. The key idea is to employ an impulsive observer coupled with an appropriate linear feedback control whenever the unmeasured subspace is stable. Otherwise, one has to employ two cascaded observers in conjunction with a linear feedback control when the unmeasured subspace is unstable. A practical stability result has been proposed to prove the stability of the closedloop systems. Simulation results on an academic example has shown good performance of the observer-based control. Future works will consist in extending the proposed results to some classes of nonlinear systems.

\section{REFERENCES}

[1] T. Raff and F. Allgower, An impulsive observer that estimates the exact state of a linear continuous-time system in predetermined finite time, Control and Automation, MED'07, 2007.

[2] G. Zheng, Y. Orlov, W. Perruquetti and J.P. Richard, Finite time observerbased control of linear impulsive systems with persistently acting impact, 18th IFAC World Congress, pp.2442-2447, 2011.

[3] E. Candes and M. B. Walkin, An introduction to compressive sampling, IEEE Signal Processing Magazine, Vol. 21, no. 2, pp. 21-30, 2008. 

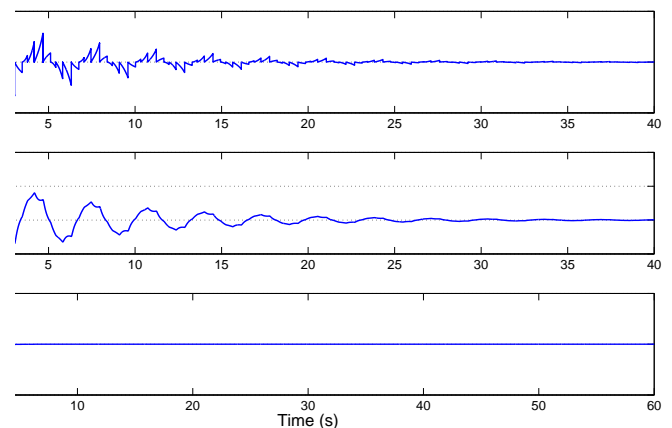

Fig. 3. The error of observation with sampling period $\theta_{k} \in[0.3 s, 0.6 s]$

[4] I. Stamova, Stability analysis of impulsive functional differential equations, De Gruyter, vol. 9, 2009.

[5] W. Haddad, V. Chellaboina and S. Neresov, Impulsive and hybrid dynamical systems: stability, dissipativity and control, Princeton University Press, 2006.

[6] L. Hettel, J. Daafouz, S. Tarbouriech and C. Prieur, Reset control systems: stabilization by nearly-periodic reset, IFAC World Congress, 2011.

[7] V. Lakshimkantham, D. Bainov and P. Simeonov, Theory of impulsive diffrential equations, World Scientific Pub Co Inc. 1989.

[8] B. Brogliato, Nonsmooth mechanics:models, dynamics and control, Springer Verlag, Ed., London, 1999.

[9] Y. Khaled, J-P. Barbot, D. Benmerzouk and K. Busawon, A new type of impulsive observer for hyperchaotic systems, IFAC Conference on Analysis and Control of Chaotic Systems, CHAOS 2012, Cancun, Mexico, 2012. 